\title{
Autophagy and p53
}

\author{
Eileen White ${ }^{1,2}$ \\ ${ }^{1}$ Rutgers Cancer Institute of New Jersey (CINJ), New Brunswick, New Jersey 08903 \\ ${ }^{2}$ Department of Molecular Biology and Biochemistry, Rutgers University, Piscataway, New Jersey 08854 \\ Correspondence: epwhite@cinj.rutgers.edu
}

\begin{abstract}
Macroautophagy (autophagy hereafter) captures, degrades, and recycles intracellular components to maintain metabolic homeostasis and protein and organelle quality control. Autophagy thereby promotes survival in starvation and prevents tissue degeneration. There is an important relationship between autophagy and p53. Autophagy suppresses p53 and also $\mathrm{p} 53$ activates autophagy. The suppression of $\mathrm{p} 53$ by autophagy is important for tumor promotion and likely also for preventing tissue degeneration. Alternatively, the activation of autophagy by p53 suggests that autophagy is part of the protective function of p53. Uncovering the underlying mechanisms of the autophagy-p53 reciprocal functional interaction and has important implications for human disease and treatment.
\end{abstract}

\begin{abstract}
Atophagy is a process controlled by the auAtophagy-related genes (Atgs), the products of which orchestrate the formation of doublemembrane vesicles that capture and sequester intracellular components (Mizushima and Komatsu 2011). These intracellular components, or autophagy cargo, include proteins, lipids, glycogen, cytoplasm, and entire organelles. Autophagosomes containing their cargo fuse with lysosomes that provide the hydrolytic enzymes to degrade the cargo. The breakdown products from cargo degradation are then released into the cytoplasm where they are recycled into metabolic and biosynthetic pathways (Rabinowitz and White 2010). Autophagy thereby has a broad effect on cellular and organismal homeostasis, including enabling survival in starvation, redox balance, and lipid, glucose, amino acid, iron, and energy homeostasis.

In tandem with the catabolic recycling activity of autophagy is its role in protein and
\end{abstract}

organelle quality control. The failure to eliminate autophagic cargo in autophagy-deficient cells and animals causes cargo accumulation that can be destructive on its own, independent of metabolic problems arising from defective recycling of cargo breakdown products (Mizushima and Komatsu 2011). Classic features of autophagy deficiency are the accumulation of proteins and protein aggregates, lipids, glycogen, and damaged mitochondria and other organelles. This failure of waste removal by autophagy greatly alters the composition of the proteome, elevates oxidative stress, promotes inflammation, and can generally alter or disrupt cellular function (Degenhardt et al. 2006; Mathew et al. 2009, 2014; Mizushima and Komatsu 2011; Deretic et al. 2013).

The products of the Atg genes are regulated by nutrients (e.g., mammalian target of rapamycin $[\mathrm{mTOR}]$ ), energy (e.g., AMP-activated protein kinase $[\mathrm{AMPK}]$ ), and stress (e.g., hyp-

Editors: Guillermina Lozano and Arnold J. Levine

Additional Perspectives on The p53 Protein available at www.perspectivesinmedicine.org

Copyright (C) 2016 Cold Spring Harbor Laboratory Press; all rights reserved; doi: 10.1101/cshperspect.a026120

Cite this article as Cold Spring Harb Perspect Med 2016;6:a026120 


\section{E. White}

oxia-inducible factors [HIFs]). In nutrient-replete conditions, basal autophagy levels are low. Starvation and a broad range of stressors dramatically induce autophagy that is critical for cell and organismal survival (Kuma et al. 2004; Mizushima et al. 2004; Komatsu et al. 2005; Karsli-Uzunbas et al. 2014). Autophagy is derepressed or activated by nutrient- and stress-signaling pathways. For example, nutrient-responsive mTOR represses autophagy. Starvation and loss of mTOR signaling derepresses inhibitory phosphorylation of the autophagy machinery to facilitate autophagy activation, catabolism, and survival. Energy crisis activates AMPK, which activates autophagy at the transcriptional and posttranscriptional level to adapt and restore metabolism, energy homeostasis, and survival. Low-oxygen conditions activate HIFs, which transcriptionally activate Atgs and autophagy to facilitate survival in hypoxia. These and other signaling pathways serve to regulate autophagy levels that are a critical component of adaptation to stress and changes in the environment.

Another critical component of stress signaling and adaptation is the tumor suppressor p53. A wide range of stressors, including DNA damage, metabolic stress, and oxidative stress, activate p53 as covered in detail in many articles in this collection. In response to these stressors, p53 regulates the transcription of genes, or acts by nontranscriptional mechanisms, to either aid in stress adaptation (e.g., cell-cycle arrest) or to eliminate cells that are beyond repair by apoptosis or senescence. A component of this p53-mediated transcriptional response is activation of autophagy (Fig. 1A). Autophagy, in turn, represses p53 levels and function (Fig. 1A). Thus, the p53 and autophagy pathways are functionally intertwined, and this has important significance to stress responses, metabolism, and cancer.

\section{AUTOPHAGY SUPPRESSES p53}

Autophagy deficiency activates the DNA damage response and promotes genome instability, suggesting that autophagy provides a protective function that suppresses DNA damage (Karantza-Wadsworth et al. 2007; Mathew et al. 2007). Conversely, autophagy promotes survival to DNA-damaging agents (Svensson et al.

A

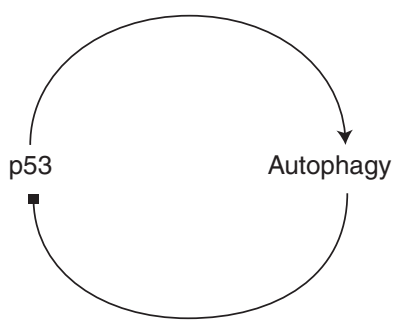

B

Autophagy $\longrightarrow$ DNA damage $\longrightarrow$ p53

C

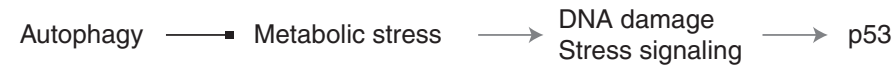

D Autophagy $\longrightarrow$ p53 $\longrightarrow \begin{aligned} & \text { Palb2-deficient } \\ & \text { tumorigenesis }\end{aligned}$

E Autophagy $\longrightarrow \begin{aligned} & \text { p53-independent } \\ & \text { mechanisms }\end{aligned} \begin{aligned} & \text { K-ras- and Braf-driven } \\ & \text { tumorigenesis }\end{aligned}$

Figure 1. Functional interactions between p53 and autophagy. See text for explanation. 
2012). The role of autophagy in suppressing DNA damage may be multifold. Autophagy defects promote use of error-prone DNA repair mechanisms (Fig. 1B) (Liu et al. 2015). Recycling by autophagy may also be necessary to provide substrates for efficient DNA replication or DNA repair, without which DNA damage is generated. In this potential scenario, recycling by autophagy supports metabolism to prevent DNA damage and thereby activation of the DNA damage response and p53 (Fig. 1C).

In mouse embryo fibroblasts, Atg7 deficiency increases p53-dependent apoptosis (Lee et al. 2012). Moreover, suppression of the DNA damage response by deficiency in Chk2 briefly delays the perinatal lethality of Atg7-deficient mice (Lee et al. 2012). Autophagy deficient mice die during physiological neonatal starvation, and force-feeding these mice only briefly delays death (Kuma et al. 2004; Komatsu et al. 2005). Neonatal mice are extremely autophagy dependent, a phenotype accentuated by nutrient limitation. As metabolic stress activates p53 to aid in adaptation (Jones et al. 2005; Maddocks et al. 2013), this suggests that autophagy may support metabolism, thereby mitigating p53 activation and cell death induction. Given these findings, it will be interesting to test whether neonatal death of autophagy-deficient mice is dependent on p53.

In contrast to neonatal mice, adult mice are less autophagy dependent under fed conditions, although they share the exquisite autophagy dependence during nutrient limitation. Acute, systemic Atg7 deletion in adult mice limits life span to 2 to 3 months, with death resulting predominantly from neurodegeneration (Karsli-Uzunbas et al. 2014). Again, whether autophagy restrains $\mathrm{p} 53$ activity or whether this neurodegeneration is p53 dependent is not known and will be interesting to test. Augmented p53 activity does underlie some brain abnormalities, neurologic dysfunction, and neurodegeneration (Trimmer et al. 1996; Morrison et al. 2003; Bae et al. 2005; Terzian et al. 2007; Checler and Alves da Costa 2014; Zhang et al. 2014), raising the possibility that restraining p53 by autophagy in this setting may be important to prevent tissue damage and organismal death.
Acute, systemic deletion of Atg7 in adult mice, although not lethal immediately under fed conditions, is lethal upon fasting (KarsliUzunbas et al. 2014). Deleting Atg7 in the whole body prevents mobilization of free fatty acids from adipose tissue, accelerates catabolism of liver glycogen, and causes muscle atrophy (cachexia) during fasting. Although serum amino acid levels are maintained likely because of elevated degradation of muscle proteins, serum glucose levels are not, and mice suffer brain damage and die from hypoglycemia. Indeed, glucose supplementation rescues death of the fasted Atg7-deleted mice (Karsli-Uzunbas et al. 2014). Whether or not augmentation of p53 function is responsible for death of fasted, Atg7-ablated mice remains to be determined.

\section{AUTOPHAGY PROMOTES CANCER BY REPRESSING p53}

In the setting of cancer, autophagy suppresses p53, significantly promoting tumorigenesis (Fig. 1D,E) (Guo et al. 2013b; White 2015). In a mouse model of hereditary breast cancer driven by loss of the Palb2 tumor suppressor, allelic loss of Atg6/Beclin1 suppresses tumor development and extends mouse survival (Huo et al. 2013). This reduction in tumorigenesis and life-span extension owing to partial Beclin1 deficiency in Palb2 loss-driven tumorigenesis is eliminated by compound $p 53$ deficiency (Huo et al. 2013). PALB2 interacts with BRCA1 and BRCA2 to promote DNA repair by homologous recombination and also suppresses oxidative stress. Loss of these functions is thought to be the mechanism by which Palb2 deficiency promotes hereditary breast cancer. p53 deficiency greatly promotes tumorigenesis with Palb2 deficiency, indicating that p53 is a barrier to tumorigenesis dependent on Palb2 loss (Huo et al. 2013). Because increased DNA damage and oxidative stress that result from Palb2 deficiency activate p53, further augmentation of p53 activation by compromised autophagy likely explains these findings (Guo et al. 2013b). This model of hereditary breast cancer shows that partial loss of autophagy is sufficient to cause p53 activation that limits tumorigenesis. 
E. White

In a mouse model of lung cancer driven by oncogenic K-ras $^{\mathrm{G} 12 \mathrm{D}}$, deficiency in Atg7 in tumor cells activates $\mathrm{p} 53$, reduces proliferation, increases cell death, and reduces tumor burden in comparison to tumors with Atg7 intact (Guo et al. 2013a). Similar findings were reported with deletion of Atg5 (Rao et al. 2014), suggesting that autophagy, not just Atg7, promotes K-ras-driven lung tumor growth. In comparison to tumors with Atg7, those without Atg7 strikingly accumulate autophagy substrates, including unprocessed LC3-I, damaged mitochondria, and p62/ SQSTM1-containing protein aggregates (Guo et al. 2013a). Thus, it is clear that Atg7 deficiency in tumors blocks autophagy, and suppresses tumor growth and promotes apoptosis.

Spontaneous activation of oncogenic K-ras in the lung of a mouse model for non-smallcell lung cancer (NSCLC) produces hyperplasia, with gradual progression to adenomas and to adenocarcinomas with acquisition of $p 53 \mathrm{mu}$ tations (Jackson et al. 2001). Deletion of Atg7 in these tumors, however, alters the course of progression from adenocarcinomas to benign oncocytomas (Guo et al. 2013a). Oncocytomas are rare, predominantly benign human neoplasms characterized by the accumulation of defective mitochondria (Gasparre et al. 2011). This raises the testable hypothesis that autophagy defects may be the underlying basis for the benign nature of these rare human tumors. If so, this would provide a natural example of how autophagy inactivation limits human cancer progression.

Despite the reduced tumor burden and benign nature of these Atg7-deficient K-ras-driven mouse lung tumors, there is no extension of life span because the mice die of pneumonia instead of cancer (Guo et al. 2013a). Autophagy deficiency commonly promotes inflammation (Degenhardt et al. 2006; Rubinsztein et al. 2015) and, in this case, the cause of the inflammation is the autophagy-deficient tumors. How autophagy-deficient tumors cause inflammation is not known but may be related to the failure to degrade components of the innate immunity pathways, increased tumor cell death, or activation of stress-induced signaling pathways (Degenhardt et al. 2006; Deretic et al. 2013; Mathew et al. 2014). It will be important to test whether the inflammatory response to autophagy-deficient tumors contributes to tumor regression.

To test whether amplified p53 induction contributes to the antitumor activity of $A \operatorname{tg} 7 \mathrm{de}-$ ficiency, $\operatorname{Trp} 53$ was deleted concurrently with activation of K-ras with or without deletion of Atg7. Trp53 loss accelerates tumorigenesis in Kras-driven lung cancer, as p53 is a barrier to tumor growth. Deletion of Atg7 still suppresses proliferation, increases apoptosis, and reduces tumor growth in the absence of $\operatorname{Tr} p 53$, although to a lesser extent than with Trp53 intact (Guo et al. 2013a,b). These findings show that Atg7 deficiency activates $\mathrm{p} 53$, which limits the growth of K-ras-driven lung cancers (Fig. 1D). Atg7loss, however, still produces antitumor activity in the absence of Trp53, indicating the existence of Trp53-independent mechanisms of tumor suppression (Fig. 1E) (Guo et al. 2013a,b). In other words, Atg7 promotes tumor growth by restraining p53 activation but also promotes tumor growth by p53-independent mechanisms.

Acute, systemic ablation of Atg7 in mice with preexisting K-ras-driven, $p 53$-deficient NSCLCs produces extinction of oncogenic signaling downstream from K-ras, massive cell death, and tumor involution, more so than that which occurs with tumor cell-specific Atg7 deletion at the time of tumor initiation (Guo et al. 2013a; Karsli-Uzunbas et al. 2014). This further shows the p53-independent tumor promotion by Atg7. These findings also suggest that host autophagy, in addition to tumor cell-autonomous autophagy, may play a role in promoting tumor growth (Karsli-Uzunbas et al. 2014).

The next question is how does Atg7 suppress p53 and promote tumorigenesis? Deletion of Atg7 in K-ras-driven, Trp53-deficient NSCLCs strikingly induces tumor lipid accumulation, which is not observed in K-ras-driven lung tumors with Trp53 intact (Guo et al. 2013a). In the absence of Trp53, Atg7 is additionally required for maintenance of mitochondrial fatty acid oxidation, suggesting that autophagy plays an important role in the metabolic adaptation to Trp53 deficiency.

How autophagy suppresses p53 activation is not known, but could be because of a reduction 
of oxidative or other stress pathways that would otherwise activate $\mathrm{p} 53$ in the absence of autophagy. It is also not known how autophagy promotes K-ras-driven NSCLC in the absence of $\mathrm{p} 53$, but it could be because of suppression of p53-independent stress pathways, particularly those responsive to metabolic crisis triggered by the absence of autophagy. Indeed, autophagy-deficient tumor cells have impaired energy charge (Guo et al. 2011). Comparison of Atg7 wild-type and deficient cell lines derived from these K-ras-driven, p53-deficient NSCLCs found that they require autophagy to survive starvation (Guo et al. 2013a). These starved Atg7-deficient tumor cells were highly dependent on glutamine for survival, suggesting that autophagy recycles protein to sustain metabolism and survival (Guo et al. 2013a,b). One can think of autophagy as conferring metabolic fitness, thereby suppressing p53 and other stressresponsive pathways that limit tumor growth. Why glutamine is so essential for the survival of autophagy-deficient tumor cells is not yet clear; however, glutamine may be critical to replenish tricarboxylic acid (TCA)-cycle intermediates because, in these glycolytic tumor cells, carbon from glucose is diverted from pyruvate to lactate and away from the TCA cycle (Guo et al. 2013a,b; Strohecker et al. 2013; Strohecker and White 2014a,b). Autophagy may supply this glutamine from protein and organelle degradation and recycling, thereby replenishing TCA-cycle intermediates (anapleurosis).

Promotion of K-ras-driven lung cancer by autophagy-mediated suppression of p53 raised the question as to whether this was Ras-specific or also generalizable to other oncogenic events. In a mouse model of lung tumorigenesis driven by oncogenic Braf ${ }^{\mathrm{V} 600 \mathrm{E}}$, Atg7 deletion initially accelerates tumor growth because of activation of the master regulator of antioxidant defense, NRF2 (Strohecker et al. 2013). NRF2 promotes the growth of lung cancers by reducing oxidative stress (DeNicola et al. 2011), suggesting that increased oxidative stress caused by deficiency in Atg7 induces a protective NRF2 response. Nonetheless, this tumor promotion by Atg7 deficiency is transient, and is followed by 553 activation, proliferative arrest, suppression of tumorigenesis, and dramatic life-span extension (Strohecker et al. 2013). p53 is a barrier to Braf-driven lung tumor growth as deletion of p53 accelerates tumorigenesis (Dankort et al. 2007). The antitumor activity of Atg7 loss is less in the absence of $p 53$ but still present (Strohecker et al. 2013). As in the case of Kras, Atg7 promotes Braf-driven lung tumor growth by limiting p53 but also does so by p53-independent mechanisms.

Cell lines derived from these Braf-driven tumors also displayed increased sensitivity to starvation and dependence on glutamine without Atg7, suggesting that mitigation of metabolic stress is the mechanism by which autophagy promotes the growth of these tumors (Strohecker et al. 2013; Strohecker and White 2014a,b). Because tumor promotion by autophagy is greater in the setting of oncogenic $\mathrm{Braf}^{\mathrm{V} 600 \mathrm{E}}$ than $\mathrm{K}$ ras ${ }^{\mathrm{G} 12 \mathrm{D}}$, this suggests that metabolic reprogramming by the mitogen-activated protein (MAP) kinase pathway elevates the requirement for autophagy (Strohecker and White 2014b).

Is the role of $A \operatorname{tg} 7$ in promoting Braf-driven tumor growth ubiquitous across tumor types or limited to lung tumors? In Braf ${ }^{\mathrm{V} 600 \mathrm{E}}$-driven and Pten heterozygous melanomas, Atg7 deletion dramatically suppresses tumor formation (Xie et al. 2015). In Braf ${ }^{\mathrm{V} 600 \mathrm{E}}$-driven and Pten-deficient melanomas, Atg7 deletion suppresses the growth and induces senescence of melanomas, producing dramatic life-span extension (Xie et al. 2015). Thus, the autophagy dependence of tumors is not related to the cell type of origin. Activation of senescence by the p53 pathway is a known barrier to melanomagenesis, but whether p53 pathway inactivation reduces the autophagy dependence of melanoma remains to be tested.

Pancreatic cancer is another example in which autophagy is important for promoting tumorigenesis. Autophagy is also up-regulated in pancreatic cancers and tumor cells (Yang et al. 2011) because of transcriptional activation of autophagy and lysosomal biogenesis (Perera et al. 2015). Moreover, autophagy deficiency in the background of K-ras ${ }^{\mathrm{G} 12 \mathrm{D}}$ activation with either $p 53$ intact or with stochastic loss of $p 53$ increases the formation of premalignant lesions (Ro- 


\section{E. White}

senfeldt et al. 2013; Yang et al. 2014). However, these premalignant lesions fail to progress to invasive cancer when autophagy is defective, which prolongs mouse survival (Rosenfeldt et al. 2013; Yang et al. 2014). Human pancreatic cancer cell lines and patient-derived xenografts with differing Trp53 mutational status are also sensitive to autophagy ablation, demonstrating that autophagy promotes the growth of pancreatic cancer (Yang et al. 2011, 2014). Although autophagy ablation with K-ras ${ }^{\text {G12D }}$ activation and deletion of both Trp53 alleles in the embryonic pancreas accelerates tumor growth (Rosenfeldt et al. 2013), this does not physiologically represent human disease (Amaravadi and Debnath 2014). Why autophagy ablation stimulates premalignant disease is not known but may be because of increased oxidative stress as seen in the Braf ${ }^{\mathrm{V} 600 \mathrm{E}}$ lung tumor model, or because of chronic tissue damage and inflammation dependent on loss of autophagy. The setting of pancreas cancer provides another example in which autophagy is required for progression from benign to malignant disease, and this again is independent of p53.

\section{MECHANISM BY WHICH AUTOPHAGY SUPPRESSES p53}

A common feature of the genetically engineered mouse models for cancer described above is identification of the requirement of autophagy for suppression of p53 and for progression from benign to malignant disease. Although the mechanisms behind these tumor-promoting functions of autophagy are unknown, important clues are emerging. Autophagy can suppress oxidative stress by eliminating reactive oxygen species (ROS)-producing organelles, such as mitochondria and peroxisomes, and also may provide metabolic substrates necessary for antioxidant defense. Oxidative stress activates p53, thus, by suppressing oxidative stress autophagy may limit p53's activity. Another possibility already mentioned is that autophagy may limit p53 activation by providing substrates for DNA replication and repair thereby preventing DNA damage. Autophagy suppresses AMPK activation by sustaining metabolism and energy homeostasis, and AMPK activates p53 (Jones et al. 2005). p53 can also be degraded by chaperone-mediated autophagy (VakifahmetogluNorberg et al. 2013). This does not explain the findings from the mouse models described here because these delete Atg5 and Atg7, which are not involved in chaperone-mediated autophagy. Compromised macroautophagy, however, may overwhelm chaperone-mediated autophagy, indirectly producing p53 accumulation. Whether these or other mechanisms control p53 levels and function to modulate cancer remain to be tested. Regardless of the mechanism, autophagy inhibitors are expected to have anticancer activity in part by functioning as p53 activators.

\section{p53 ACTIVATES THE TRANSCRIPTION OF AUTOPHAGY-RELATED GENES}

The above studies show that autophagy suppresses p53 and there is also evidence that p53 activates autophagy (Fig. 1A). p53 is a sequence-specific DNA-binding transcription factor and, among the many genes regulated directly by p53, are autophagy genes. One p53 target gene is damage-regulated autophagy modulator (Dram), and induction of DRAM expression promotes autophagy (Crighton et al. 2006). DRAM appears to be required for apoptosis induction by $\mathrm{p} 53$, although the mechanism is unclear (Crighton et al. 2006). Another p53 target gene that promotes autophagy is Isg20L1 (Eby et al. 2010). Isg20L1 overexpression promotes autophagy and cell death, which was partly rescued by Atg5 deficiency. Using a more systematic approach, p53 target genes induced by DNA damage were identified by chromatin immunoprecipitation sequencing and RNA sequencing. Among the genes directly activated by p53 are a suite of autophagy genes, including Ulk1 and Atg7 (Kenzelmann Broz et al. 2013). p53 induction in this setting induces autophagy. The functional significance of p53-induced autophagy revealed stimulation of apoptosis in response to DNA damage and suppression of transformation by E1A and H-ras (Kenzelmann Broz et al. 2013). Given the global and context-dependent role of autophagy, going forward it will be important to 
Autophagy and p53

functionally address p53 transcription-mediated autophagy in physiological conditions in vivo. For example, would autophagy wild-type and deficient mice respond differently to DNA damage? If there is a differential response, would that be altered by the absence of p53? With the development of switchable mouse models for conditional, systemic autophagy inactivation in vivo (Karsli-Uzunbas et al. 2014), addressing these questions is now possible.

In contrast to the direct transcriptional activation of autophagy by p53, p53 deficiency activates autophagy that promotes survival (Tasdemir et al. 2008). The absence of p53 may create stress, requiring remediation by autophagy. This too may be clarified by deleting an essential autophagy gene in mice with or without compound deletion of p53 to address the functional outcome. Nontranscriptional mechanisms by which p53 can regulate autophagy have also been reported (Tasdemir et al. 2008; Xiao et al. 2015), and it will be interesting to determine the physiologic context, in which these events may be important.

\section{CONCLUDING REMARKS}

In summary, cellular self-cannibalization by autophagy provides metabolic substrates that promote survival in starvation. Autophagy is also critical for protein and organelle quality control that maintains tissue and organismal homeostasis. In normal tissues, it will be important to address whether autophagy suppresses p53 activation, which contributes to the destructive consequences of autophagy deficiency. In genetically engineered mouse models of cancer, it is clear that autophagy suppresses p53 activation and subsequent p53-dependent proliferative arrest, apoptosis, and senescence, which limits tumorigenesis (White 2015). Suppression of p53 by autophagy is an important mechanism of tumor promotion that otherwise limits tumorigenesis to benign disease. Autophagy additionally promotes tumorigenesis by p53-independent mechanisms that remain to be identified. p53 also activates autophagy, which may be a feedback mechanism to control p53 function in the setting of adaptation to metabolic stress.

\section{ACKNOWLEDGMENTS}

E.W. acknowledges support from NIH Grants (R01 CA163591, R01 CA130893, and R01 CA188096), the Val Skinner Foundation, and the Rutgers Cancer Institute of New Jersey (CINJ) (P30 CA72720). E.W. is an advisor to Forma Therapeutics.

\section{REFERENCES}

Amaravadi R, Debnath J. 2014. Mouse models address key concerns regarding autophagy inhibition in cancer therapy. Cancer Discov 4: 873-875.

Bae BI, Xu H, Igarashi S, Fujimuro M, Agrawal N, Taya Y, Hayward SD, Moran TH, Montell C, Ross CA, et al. 2005. p53 mediates cellular dysfunction and behavioral abnormalities in Huntington's disease. Neuron 47: 29-41.

Checler F, Alves da Costa C. 2014. p53 in neurodegenerative diseases and brain cancers. Pharmacol Ther 142: 99-113.

Crighton D, Wilkinson S, O’Prey J, Syed N, Smith P, Harrison PR, Gasco M, Garrone O, Crook T, Ryan KM. 2006 Dram, a p53-induced modulator of autophagy, is critical for apoptosis. Cell 126: 121-134.

Dankort D, Filenova E, Collado M, Serrano M, Jones K, McMahon M. 2007. A new mouse model to explore the initiation, progression, and therapy of brafv600e-induced lung tumors. Genes Dev 21: 379-384.

Degenhardt K, Mathew R, Beaudoin B, Bray K, Anderson D, Chen G, Mukherjee C, Shi Y, Gelinas C, Fan Y, et al. 2006. Autophagy promotes tumor cell survival and restricts necrosis, inflammation, and tumorigenesis. Cancer Cell 10: $51-64$.

DeNicola GM, Karreth FA, Humpton TJ, Gopinathan A, Wei C, Frese K, Mangal D, Yu KH, Yeo CJ, Calhoun ES, et al. 2011. Oncogene-induced nrf2 transcription promotes ros detoxification and tumorigenesis. Nature 475: 106-109.

Deretic V, Saitoh T, Akira S. 2013. Autophagy in infection, inflammation and immunity. Nat Rev Immunol 13: 722737.

Eby KG, Rosenbluth JM, Mays DJ, Marshall CB, Barton CE, Sinha S, Johnson KN, Tang L, Pietenpol JA. 2010. Isg2011 is a p53 family target gene that modulates genotoxic stress-induced autophagy. Mol Cancer 9: 95.

Gasparre G, Romeo G, Rugolo M, Porcelli AM. 2011. Learning from oncocytic tumors: Why choose inefficient mitochondria? Biochim Biophys Acta 1807: 633-642.

Guo JY, Chen HY, Mathew R, Fan J, Strohecker AM, KarsliUzunbas G, Kamphorst JJ, Chen G, Lemons JM, Karantza $\mathrm{V}$, et al. 2011. Activated ras requires autophagy to maintain oxidative metabolism and tumorigenesis. Genes Dev 25: $460-470$.

Guo JY, Karsli-Uzunbas G, Mathew R, Aisner SC, Kamphorst JJ, Strohecker AM, Chen G, Price S, Lu W, Teng $\mathrm{X}$, et al. 2013a. Autophagy suppresses progression of k-ras-induced lung tumors to oncocytomas and maintains lipid homeostasis. Genes Dev 27: 1447-1461. 
E. White

Guo JY, Xia B, White E. 2013b. Autophagy-mediated tumor promotion. Cell 155: 1216-1219.

Huo Y, Cai H, Teplova I, Bowman-Colin C, Chen G, Price S, Barnard N, Ganesan S, Karantza V, White E, et al. 2013. Autophagy opposes p53-mediated tumor barrier to facilitate tumorigenesis in a model of palb2-associated hereditary breast cancer. Cancer Discov 3: 894-907.

Jackson EL, Willis N, Mercer K, Bronson RT, Crowley D, Montoya R, Jacks T, Tuveson DA. 2001. Analysis of lung tumor initiation and progression using conditional expression of oncogenic k-ras. Genes Dev 15: 3243-3248.

Jones RG, Plas DR, Kubek S, Buzzai M, Mu J, Xu Y, Birnbaum MJ, Thompson CB. 2005. Amp-activated protein kinase induces a p53-dependent metabolic checkpoint. Mol Cell 18: 283-293.

Karantza-Wadsworth V, Patel S, Kravchuk O, Chen G, Mathew R, Jin S, White E. 2007. Autophagy mitigates metabolic stress and genome damage in mammary tumorigenesis. Genes Dev 21: 1621-1635.

Karsli-Uzunbas G, Guo JY, Price S, Teng X, Laddha SV, Khor S, Kalaany NY, Jacks T, Chan CS, Rabinowitz JD, et al. 2014. Autophagy is required for glucose homeostasis and lung tumor maintenance. Cancer Discov 4: 914-927.

Kenzelmann Broz D, Spano Mello S, Bieging KT, Jiang D, Dusek RL, Brady CA, Sidow A, Attardi LD. 2013. Global genomic profiling reveals an extensive p53-regulated autophagy program contributing to key p53 responses. Genes Dev 27: 1016-1031

Komatsu M, Waguri S, Ueno T, Iwata J, Murata S, Tanida I, Ezaki J, Mizushima N, Ohsumi Y, Uchiyama Y, et al. 2005. Impairment of starvation-induced and constitutive autophagy in atg7-deficient mice. J Cell Biol 169: 425-434.

Kuma A, Hatano M, Matsui M, Yamamoto A, Nakaya H, Yoshimori T, Ohsumi Y, Tokuhisa T, Mizushima N. 2004. The role of autophagy during the early neonatal starvation period. Nature 432: 1032-1036.

Lee IH, Kawai Y, Fergusson MM, Rovira II, Bishop AJ, Motoyama N, Cao L, Finkel T. 2012. Atg7 modulates p53 activity to regulate cell cycle and survival during metabolic stress. Science 336: 225-228.

Liu EY, Xu N, O'Prey J, Lao LY, Joshi S, Long JS, O’Prey M, Croft DR, Beaumatin F, Baudot AD, et al. 2015. Loss of autophagy causes a synthetic lethal deficiency in DNA repair. Proc Natl Acad Sci 112: 773-778.

Maddocks OD, Berkers CR, Mason SM, Zheng L, Blyth K, Gottlieb E, Vousden KH. 2013. Serine starvation induces stress and p53-dependent metabolic remodelling in cancer cells. Nature 493: 542-546.

Mathew R, Kongara S, Beaudoin B, Karp CM, Bray K, Degenhardt K, Chen G, Jin S, White E. 2007. Autophagy suppresses tumor progression by limiting chromosomal instability. Genes Dev 21: 1367-1381.

Mathew R, Karp CM, Beaudoin B, Vuong N, Chen G, Chen HY, Bray K, Reddy A, Bhanot G, Gelinas C, et al. 2009. Autophagy suppresses tumorigenesis through elimination of p62. Cell 137: 1062-1075.

Mathew R, Khor S, Hackett SR, Rabinowitz JD, Perlman DH, White E. 2014. Functional role of autophagy-mediated proteome remodeling in cell survival signaling and innate immunity. Mol Cell 55: 916-930.
Mizushima N, Komatsu M. 2011. Autophagy: Renovation of cells and tissues. Cell 147: 728-741.

Mizushima N, Yamamoto A, Matsui M, Yoshimori T, Ohsumi Y. 2004. In vivo analysis of autophagy in response to nutrient starvation using transgenic mice expressing a fluorescent autophagosome marker. Mol Biol Cell 15: $1101-1111$.

Morrison RS, Kinoshita Y, Johnson MD, Guo W, Garden GA. 2003. p53-dependent cell death signaling in neurons. Neurochem Res 28: 15-27.

Perera RM, Stoykova S, Nicolay BN, Ross KN, Fitamant J, Boukhali M, Lengrand J, Deshpande V, Selig MK, Ferrone CR, et al. 2015. Transcriptional control of autophagylysosome function drives pancreatic cancer metabolism. Nature 524: 361-365.

Rabinowitz JD, White E. 2010. Autophagy and metabolism. Science 330: 1344-1348.

Rao S, Tortola L, Perlot T, Wirnsberger G, Novatchkova M, Nitsch R, Sykacek P, Frank L, Schramek D, Komnenovic $\mathrm{V}$, et al. 2014. A dual role for autophagy in a murine model of lung cancer. Nat Commun 5: 3056.

Rosenfeldt MT, O’Prey J, Morton JP, Nixon C, MacKay G, Mrowinska A, Au A, Rai TS, Zheng L, Ridgway R, et al. 2013. p53 status determines the role of autophagy in pancreatic tumour development. Nature 504: 296-300.

Rubinsztein DC, Bento CF, Deretic V. 2015. Therapeutic targeting of autophagy in neurodegenerative and infectious diseases. J Exp Med 212: 979-990.

Strohecker AM, White E. 2014a. Autophagy promotes brafv600e-driven lung tumorigenesis by preserving mitochondrial metabolism. Autophagy 10: 384-385.

Strohecker AM, White E. 2014b. Targeting mitochondrial metabolism by inhibiting autophagy in braf-driven cancers. Cancer Discov 4: 766-772.

Strohecker AM, Guo JY, Karsli-Uzunbas G, Price SM, Chen GJ, Mathew R, McMahon M, White E. 2013. Autophagy sustains mitochondrial glutamine metabolism and growth of brafv600e-driven lung tumors. Cancer Discov 3: $1272-1285$.

Svensson JP, Fry RC, Wang E, Somoza LA, Samson LD. 2012. Identification of novel human damage response proteins targeted through yeast orthology. PloS ONE 7: e37368.

Tasdemir E, Maiuri MC, Galluzzi L, Vitale I, DjavaheriMergny M, D’Amelio M, Criollo A, Morselli E, Zhu C, Harper F, et al. 2008. Regulation of autophagy by cytoplasmic p53. Nat Cell Biol 10: 676-687.

Terzian T, Wang Y, Van Pelt CS, Box NF, Travis EL, Lozano G. 2007. Haploinsufficiency of $\mathrm{mdm} 2$ and $\mathrm{mdm} 4$ in tumorigenesis and development. Mol Cell Biol 27: 5479-5485.

Trimmer PA, Smith TS, Jung AB, Bennett JP Jr. 1996. Dopamine neurons from transgenic mice with a knockout of the p53 gene resist MPTP neurotoxicity. Neurodegeneration 5: 233-239.

Vakifahmetoglu-Norberg H, Kim M, Xia HG, Iwanicki MP, Ofengeim D, Coloff JL, Pan L, Ince TA, Kroemer G, Brugge JS, et al. 2013. Chaperone-mediated autophagy degrades mutant p53. Genes Dev 27: 1718-1730.

White E. 2015. The role for autophagy in cancer. J Clin Invest 125: $42-46$.

Xiao J, Zhang T, Xu D, Wang H, Cai Y, Jin T, Liu M, Jin M, Wu K, Yuan J. 2015. Fbxl20-mediated vps34 ubiquitina- 
tion as a p53 controlled checkpoint in regulating autophagy and receptor degradation. Genes Dev 29: 184-196.

Xie X, Koh JY, Price S, White E, Mehnert JM. 2015. Atg7 overcomes senescence and promotes growth of brafv600e-driven melanoma. Cancer Discov 5: 410-423.

Yang S, Wang X, Contino G, Liesa M, Sahin E, Ying H, Bause A, Li Y, Stommel JM, Dell'antonio G, et al. 2011. Pancreatic cancers require autophagy for tumor growth. Genes Dev 25: 717-729.
Yang A, Rajeshkumar NV, Wang X, Yabuuchi S, Alexander BM, Chu GC, Von Hoff DD, Maitra A, Kimmelman AC. 2014. Autophagy is critical for pancreatic tumor growth and progression in tumors with p53 alterations. Cancer Discov 4: 903-913.

Zhang Y, Xiong S, Li Q, Hu S, Tashakori M, Van Pelt C, You MJ, Pageon L, Lozano G. 2014. Tissue-specific and age-dependent effects of global mdm2 loss. J Pathol 233: $380-391$. 


\title{
$\&_{\mathrm{CSH}}^{\infty} \&$ Cold Spring Harbor

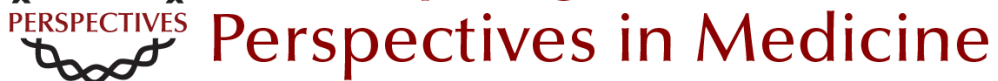

\section{Autophagy and p53}

\author{
Eileen White
}

Cold Spring Harb Perspect Med 2016; doi: 10.1101/cshperspect.a026120

Subject Collection The p53 Protein

\section{Targeting the MDM2-p53 Protein-Protein Interaction for New Cancer Therapy: Progress and Challenges \\ Shaomeng Wang, Yujun Zhao, Angelo Aguilar, et al.}

Structural Evolution and Dynamics of the p53

Proteins

Giovanni Chillemi, Sebastian Kehrloesser,

Francesca Bernassola, et al.

Exploiting the p53 Pathway for Therapy Chit Fang Cheok and David Philip Lane

The Regulation of Cellular Functions by the p53

Protein: Cellular Senescence

Crystal A. Tonnessen-Murray, Guillermina Lozano and James G. Jackson

The Transactivation Domains of the p53 Protein Nitin Raj and Laura D. Attardi

The Evolution of the Ribosomal Protein-MDM2p53 Pathway

Chad Deisenroth, Derek A. Franklin and Yanping Zhang

Somatic TP53 Mutations in the Era of Genome

Sequencing

Pierre Hainaut and Gerd P. Pfeifer

The Paradox of p53: What, How, and Why?

Yael Aylon and Moshe Oren
Control of Cellular Aging, Tissue Function, and Cancer by p53 Downstream of Telomeres Caitlin M. Roake and Steven E. Artandi

Inherited TP53 Mutations and the $\mathbf{L i}-$ Fraumeni Syndrome

Tanya Guha and David Malkin

TP53 Mutations in Hypodiploid Acute Lymphoblastic Leukemia

Evan Q. Comeaux and Charles G. Mullighan

Transcriptional Regulation by Wild-Type and

Cancer-Related Mutant Forms of p53

Neil T. Pfister and Carol Prives

The Inherited p53 Mutation in the Brazilian

Population

Maria Isabel Achatz and Gerard P. Zambetti

TP53 Mutations in Breast and Ovarian Cancer Laxmi Silwal-Pandit, Anita Langerød and Anne-Lise Børresen-Dale

p53 and the Carcinogenicity of Chronic Inflammation

Andrei V. Gudkov and Elena A. Komarova

Oncogenic Mutant p53 Gain of Function

Nourishes the Vicious Cycle of Tumor

Development and Cancer Stem-Cell Formation

Yoav Shetzer, Alina Molchadsky and Varda Rotter

For additional articles in this collection, see http://perspectivesinmedicine.cshlp.org/cgi/collection/ 